\title{
MAGNETIC RESONANCE IMAGING ASSESSMENT OF THICKENED LIGAMENTUM FLAVUM IN DEGENERATIVE LUMBAR SPINE-CORELATION WITH AGE, GENDER, DEGENERATIVE DISC, RIGHT-LEFT ASYMMETRY IN CENTRAL INDIA POPULATION
}

\author{
Vijaya Rajesh Kamble
}

\author{
NKPSIMS and Research Centre, India
}

\section{A R T I C L E I N F O}

\section{Article History:}

Received $10^{\text {th }}$ December, 2016

Received in revised form $9^{\text {th }}$ January, 2017

Accepted $12^{\text {th }}$ February, 2017

Published online $28^{\text {th }}$ March, 2017

\section{Key words:}

Ligamentum flavum thickness, degenerative, Magnetic Resonance Imaging, gender, disc degeneration, asymmetry. \begin{abstract}
A B S T R A C T
Background: Thickened ligamentum flavum(LF) has been traditionally related to lumbar canal stenosis. Till date many studies has been done to evaluate thickened LF and its association with age, gender, disc degeneration, mean LF thickness, increment in LF thickness at lumbar levels and right-left asymmetry or side dominance. But many discrepancies were found. Present study aimed to study these associations in the central India population.

Material and methods: 345 individuals of 20 to 70 years old, with complains of low back ache and/ or leg pain whose Magnetic Resonance Imaging (MRI)of lumbosacral spine was done, were studied retrospectively. LF thickness was measured on axial T1WI of MRI at L3-4,L4-5 and L5-S1 levels. Mean and standard deviations of the parameters and t tests were taken. Significant difference was set at $\mathrm{P}<0.05$.

Results: LF thickness significantly increased with age at all levels and was more thicker in females than males. Increment was larger at L4-5 than at L5-S1 level. Increased LF thickness was observed with type IV $-\mathrm{V}$ disc degeneration, but was not statistically significant $(\mathrm{p}>0.05)$. Mean LF thickness at L5-S1 level on right side was found to be more i.e $4.56 \mathrm{~mm}$ followed by L4-5(right-4.36, left-3.70mm) and L3-4 level(right-3.63 mm ,left$2.86 \mathrm{~mm}$ ). Mean thickness at L5-S1level on left side was found to be less i.e $1.28 \mathrm{~mm}$. Significant asymmetry was found between LF thickness on right and left side at each lumbar level and right sided dominance was found at each level.

Conclusion: LF thickness is an age and gender dependent phenomenon. Right sided dominance is found at each lumbar level. LF thickness is increased at L5-S1 level and L4-5 levels.
\end{abstract}

Copyright $(2017$ Vijaya Rajesh Kamble. This is an open access article distributed under the Creative Commons Attribution License, which permits unrestricted use, distribution, and reproduction in any medium, provided the original work is properly cited.

\section{INTRODUCTION}

The ligamentum flavum originates from the anterior aspect of the lower margin of one lamina and inserts on the lamina below along its posterior surface .' Ligamentum flavum changes its appearance on sagittal MRI from midline to parasagittal scan. ${ }^{2}$ It is seen as an oblique, linear, low signal intensity band that attaches to the superior border of one spinous process and the inferior surface of the spinous process below it. On parasagittal scans, LF is seen as an inhomogenous triangle with broader base near the lamina and narrow base inferiorly (fig $1 \mathrm{a}$ and $\mathrm{b}$ ). On axial MR studies, the LF appears as a V-shaped structure that covers the laminae and facet joint anteriorly. LF is similar to signal intensity of muscle.

*Corresponding author: Vijaya Rajesh Kamble

NKPSIMS and Research Centre, India
But the signal intensity can be variable on MR because ligamentum flavum undergoes age related degenerative changes or become infiltrated with fat. Rarely it may undergo ossification or calcification. The elasticity of LF decreases with age.

As a consequence to hypertrophy of ligamentum flavum, diameter of the spinal canal gets reduced posteriorly. This reduced diameter of the spinal canal may notably squeeze the dural sac and nerve roots resulting in symptoms and these symptoms are strangely without any herniated disc, disc bulge or disc protrusion. ${ }^{3}$ Whenever there is increase in the size of $\mathrm{LF}$, a posterior protrusion of the disc or a combination of the two, they causes nerve root compression. Hypertrophy of the ligament can be unilateral or bilateral and results from either injury or scar tissue. Normal ligaments are composed entirely of yellow elastic fibres and grossly have considerable elasticity. It appears that during injury rupture of some of the elastic fibres of the ligamentum flava occurs, causing them to expand. Eventually repair takes place and results in scar tissue 
formation with further hypertrophy of the ligaments, resulting in compression of the nerve roots. ${ }^{4}$

This study is aimed to provide details of LF thickness on either side at lumbar levels L3-4, L4-5 and L5-S1 and its correlation with age, gender, type of disc degeneration, side asymmetry and to determine the correlation between LF thickening and increasing age.

\section{MATERIAL AND METHODS}

Prior approval of Institutional Ethical committee board was taken before the start of the study. This was a 12 month, retrospective study assessing the spinal magnetic resonance imaging (MRIs) of 345 adult individuals with complains of low back ache from 20 to 70 years old. Total 2,070 ligamenta flava were measured from the database of department of Radiodiagnosis. Data was divided into three groups according to the patients age: $20-40$ years; $41-60$ years and more than 60 years. Patients with history of previous lumbar surgery, radiotherapy, congenital anamolies, scoliosis, spondylolisthesis were excluded. Also patients with cardiac pacemakers, aneurysmal clips and metallic joint implants were excluded from the study.

The included patients underwent MRI study of whole spine on a 1.5 Tesla MRI Scanner (General Electronics medical systems Signa HD XT 16 Channel MRI).

In each patient the MRI was performed in the sagital and axial plane. The pasted view was used to count the number of vertebrae and to find the cases of lumbarisation or sacralisation. These cases were than marked separately.

T2Weighted sagittal images were used to locate the spinal level of the intervertebral spaces and after confirmation, measurement of the LF thickness were made on the T1 weighted axial image at the L3-4,L4-5 and L5-S1 spinal levels. These measurements were taken at Facet joint level (fig 2). Axial TIWI's were used because as compared to T2Weighted images, T1WI gave better delineation of the LF and facet joints. The measurements were done with the help of tools panel software installed on work station.

The maximum thickness of the LF was measured on both right and left sides at the spinal levels L3-4,L4-5 and L5S1.To minimize error, the average of three readings was taken.

Degree of lumbar disc degeneration was classified according to Pfirrmman et al. ${ }^{5}$ Assessment of disc degeneration was done on T2Weighted sagittal imaging. Disc degeneration was categorised into two groups as type I-III and type IV $-\mathrm{V}$. The LF thickness and these types of disc degeneration were compared statistically.

Statistical evaluation was done using $\mathrm{R}$ version 3.2.0. LF thickness association with age, gender, disc degeneration, lumbar level and side dominance were studied. Mean and standard deviations of the study parameters were calculated. These different associations were studied and was considered to be statistically significant when the probability value was less than 0.05. $(P \leq 0.05)$

\section{RESULTS}

Total three hundred forty five patients participated in the study. Mean age was 44.1 years \pm 13.58 years. $173(50.14 \%)$ males and 172 females(49.85\%) took part in this study. Male to female ratio was $1: 1$. Rest of the results are shown in the tables 1-6.

\section{DISCUSSION}

In the present study, LF thickness increased significantly with age at all levels( $\mathrm{P}$ value $<0.05)$. As the age increased, LF thickness also increased. Statistically significant difference was found between LF thickness and different age groups. This agrees with other studies . $.7,8,9,10,11$

Altinkaya et $a l^{6}$ and Sairyo et $a l^{7}$ found that LF thickness at all levels increased significantly with age. They did this assessment on Magnetic resonance imaging. Sairyo et al ${ }^{7}$ stated that increased LF thickness can already be seen in younger age group of 20-30 years.

Sakamaki et al $^{8}$ also observed that LF thickness increased with age. They observed that in 20-29 years of age group, LF was over $3.0 \mathrm{~mm}$ at L4-5 level and in many of these subjects it was more than $3.5 \mathrm{~mm}$ thick. Also they said that thickening of LF at L4-5 has already started in subjects of 30-39years of age group. They concluded that thickening of LF was not due to the buckling of LF into the spinal canal with disc degeneration.

In Abbas et al study ${ }^{9}$, significant changes in LF thickness were observed after the age of 40 years. It was also observed that 22 individuals of $<30$ years of age $(4.5 \%$ to $13.6 \%$ of these subjects) showed LF thickness of more than $4 \mathrm{~mm}$ (depending on the vertebral level). Akreyi et al ${ }^{10}$ found that LF thickness increased proportionately with age.

In a study by Kolte et al ${ }^{11}$, LF thickness at L4-5 and L5-S1 levels was significantly increased with age. LF thickness $>$ $4 \mathrm{~mm}$ was found in significant number of subjects of young age group (20 to 40 years), however prevalence was not calculated. They suggested the role of mechanical stress in the increased LF thickness in young adult population. Role of mechanical stress for the increased LF thickness was also suggested by Safak et $a l^{3}$.

Sairyo et al $^{7}$ studied the pathomechanism of LF hypertrophy based on clinical, biomechanical, histologic and biologic assessments and concluded that fibrosis was the main cause of LF hypertrophy and fibrosis is caused by the accumulation of mechanical stress with the aging process.

However in the studies by Safak et $a l^{3}$ and Fukuyama et al ${ }^{12}$, no association was found between LF thickness and increasing age.

In the present study, LF thickness increased at all levels i.e at L3-4, L4-5 and L5-S1 levels with increase in age.

In the present study, LF thickness of $>4 \mathrm{~mm}$ was found in younger age group i.e 9-40 years of age at L5-S1 and L4-5 levels. This is in close agreement with Sairyo et al ${ }^{7}$, Abbas et $a l^{9}$ and Kolte et al . ${ }^{11}$

Sairyo et al $^{7}$ and Sakamaki et al ${ }^{8}$, stated that the increments at L4-5 and L3-4 levels were larger than the increments at L2- 
3 and L5-S1levels. Also Altinkaya et $a l^{6}$ and Kolte et al ${ }^{11}$ found the increments to be larger at L4-5 than at L5S1level.This agrees with the present study, where increment was observed to be larger at L4-5 level than at L5-S1 level.

The LF thickness was found to be more in females than males at all levels. Significant difference was found in LF thickness with respect to gender $(p<0.05)$ except at L3-4 level on left side. This finding agreed with Akreyi et al ${ }^{10}$ whearas in Safak et $a l^{3}$ and Abbas et al ${ }^{9}$ no such gender dependence was found. This finding could be attributed to the kind of hard work like farming and heavy labour work done by females in this central part of India.

Relatively increased LF thickness was observed with type IV $-\mathrm{V}$ disc degeneration. But this was not statistically significant $(\mathrm{p}>0.05)$. This result agreed with Akreyi et al. ${ }^{10}$ In Akreyi et $a l^{10}$, significant difference was found at L2-3 level, but this disc level was not included in the present study.

In Sakamaki et al study ${ }^{8}$, no corelation was found between thickness of LF and decrease of the disc height (disc degeneration) in elderly patients. They concluded that LF thickening was not the buckling of LF into the spinal canal with disc degeneration. Whearas in Altinkaya et al study ${ }^{6}$, increased LF thickness was found with type IV-V degeneration at all levels as compared to patients with grade I-III disc degeneration.

Okuda et $a l^{13}$ studied the pathogenesis of ligamentum flavum in degenerative lumbar disease and observed that calcification was frequently seen in elderly patients, ossification was associated with greater percentage slip in spondylolisthesis and chondroid cells were observed in patients with spondylolisthesis.

The mean of LF thickness in the present study were, at L3-4 level: right-3.63mm ,left-2.86mm; L4-5 level: right-4.36 ,left$3.70 \mathrm{~mm}$; L5-S1level: right-4.56mm, left-1.28mm.(Table$6)$.In comparison with the other imaging based studies carried out in the living subjects,(table 7) mean LF thickness in the present study at L4-5 level(Rt-4.36mm ; Lt-3.70mm)is found to be relatively in close agreement with Grenier et al ${ }^{14}$ (mean LF thickness-5mm), Park et al ${ }^{15}$ (4.44mm), Abbas et al ${ }^{9}$ (Rt$3.96 \mathrm{~mm}$; Lt-3.90mm), Safak et al ${ }^{3}$ (Rt-3.40mm , Lt-3.46 $\mathrm{mm}$ ), Altinkaya et al $^{6}$ (mean LF thickess-3.85), Kolte et al ${ }^{11}$ (Rt-3.7 mm;Lt-3.8 mm). Chokshi et al ${ }^{16}$ and Fukuyama et $a l^{12}$ found the mean LF thickness to be $4.09-5.03 \mathrm{~mm}$ and $4.0 \pm 1.1 \mathrm{~mm}$ respectively, in subjects with degenerative disease which is in close agreement with the present study.

Mean LF thickness of the present study at the different lumbar levels was also compared with the other cadaveric and surgical based studies. Mean LF thickness in the present study is found to be closely resembling Spurling et al ${ }^{17}$ (L3-4level, 4.3mm : L4-5level,4.4mm; L5-S1level ,4.2 mm), Horwitz et al 18 (L3-4level, $3.5 \mathrm{~mm}$;L4-5level,3.8mm; L5-S1level ,3.6mm).Except in the present study the thickness at L5-S1 level is on a higher side(L5-S1- right $-4.56 \mathrm{~mm}$ ) and do not resemble with the respective level in Horwitz et al ${ }^{18}$. Contrary to this, Ramani et al ${ }^{19}$ reported much higher values (L4-5 level-6.13 mm: L5-S1level- $5.2 \mathrm{~mm}$ ). Abdel-Meguid et $a l^{20}$ also carried out a cadaveric study on 14 normal cadaver lumbar vertebral columns and he found the mean thickness to be $4.5 \pm 0.8 \mathrm{SD}$. He also studied computed tomography of 30 normal living subjects at L3-4 level in the same setting and found mean thickness at L3-4 level to be $4.3 \pm 0.6 \mathrm{SD}$.In this study, the subjects selected were not having any form of degenerative lumbar disease. He observed that the ligament flava was slightly wider and thicker in its measurement on cadavers as compared to the CT measurements on the living subjects.

So it can be concluded that the discrepancies between the cadaveric study and living subjects imaging based studies, more or less will be always there and hence comparisons are to be made between the respective standard modalities and subjects.

In comparison with other studies, the mean LF thickness at L5-S1 level in the present study was found to be on the higher side i.e L5-S1 -Right side is $4.56 \mathrm{~mm}$ whearas the mean LF thickness on left side was found to be $1.28 \mathrm{~mm}$ which is very low. This could be attributed to more mechanical stress on the right side at L5-S1 level.

Present study showed that mean LF thickness was more at L5S1 level followed by L4-5 level and than L3-4 level. This observation was consistent with Safak et $a l^{3}$ and Fukuyama et $a l^{12}$. Safak et $a l^{3}$ attributed this observation to the increased mechanical stress at this level.

Kolte et al ${ }^{11}$ and Akreyi et al ${ }^{10}$ found that the mean LF thickness was more at L4-5 followed by L5-S1 and L3-4 whearas in Sairyo et $a l^{7}$ thickest LF was found at L4-5 level. In Abbas et al study ${ }^{9}$ and Altinkaya et al study ${ }^{6}$, thickest LF was found at L4-5 followed by L3-4 and than L5-S1.They related this to the hypermobility of spine at these two levels i.e L4-5 and L3-4.

In the present study, LF thickness on right and left side at each lumbar level is also compared (table 6) and right LF was found to be thicker than left one at each spinal level studied. This difference was found to be statistically significant ( $p$ value $<0.05$ ). This observation probably suggest the possible role of degree of asymmetry and instability.

Abbas et al ${ }^{9}$ also found significant asymmetry in LF thickness at L3-4 and L5-S1levels, with more ligamentum flavum thickness on right side as compared to the left side and $\mathrm{P}$ value $<0.05$ (significant). He attributed this observation to the right thoracic built -in rotation in non scoliosis spine at the mid and lower thoracic vertebrae [21]. He assumed that there should be a compensatory rotation to the left of the lumbar spine that increases the tension forces in the right spine complex, including the right sided LF, leading eventually to a more thickening of the right LF. ${ }^{9}$

Safak et $\mathrm{al}^{3}$ also found a statistically significant difference in LF thickness between contralateral sides but found the left sided LF to be thicker than right side. He explained this observation on the basis of asymmetrical mechanical stress that the LF has to carry in the lifetime which may be responsible for asymmetrical thickening. They also suggested that this asymmetry could be the effect of individual side

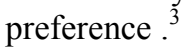

The present study suggests a strong presence of right side dominance. 

corelation with age, gender, degenerative disc, right-left asymmetry in central india population

\section{CONCLUSION}

LF thickness is an age dependent event and appears to increase with increasing age. It means that as the age increases degeneration occurs and LF thickness also increases. But many subjects of the young age group (20-40 years) also showed LF thickness $>4 \mathrm{~mm}$. LF thickness is a gender dependent event and found to be more in females. Right sided dominance of LF thickness at all lumbar spinal levels is noted.

Ethical standards: The study was approved by the Institutional ethical committee.

Conflict of interest: The authors whose names are listed on this paper certify that they have no affiliations with or involvement in any organisation or entity with any financial interest or non financial interest in the subject mater or material discussed in this manuscript.

\section{References}

1. Dorwart RH, Sauerland EK, Haughton VM et al. Normal lumbosacral spine. In Newton TH, Pitts DG, editors: Computed Tomography of the spine and spinal cord. san Anselmo, Clavadel Press 1983 pp.93-113.

2. Sze G, Bravo S, Baierl P, SHIMKIN PM .Developing spinal column: gadolinium enhanced MR imaging. Radiol 1991;180:497-502.

3. Safak AA, Is M, Sevinc O, et al. The thickness of the ligamentum flavum in relation to age and gender. Clin Anatomy $2010 ; 23: 79-83$.

4. Brown HA. Enlargement of the ligamentum flavum. $J$ Bone Joint Surg Am 1938;20:325-38.

5. Pfirrmann CW, Metzdorf A, Zanetti M, Hodler J, Boos N. Magnetic resonance classification of lumber intervertebral disc degeneration. Spine 2001; 26(17): 1873-8.

6. Altinkaya N, Yildirim T, Demir S, Alkan O, Sarica FB. Factors associated with the thickness of the ligamentum flavum: is ligamentum flavum thickening due to hypertrophy or buckling? Spine (Phila Pa 1976) 2011;36:E1093-7.

7. Sairyo K, Biyani A, Goel V, et al. Pathomechanism of ligamentum flavum hypertrophy: a multidisciplinary investigation based on clinical, biomechanical, histologic, and biologic assessments. Spine (Phila Pa 1976) 2005;30:2649-56.

8. Sakamaki T, Sairyo K, Sakai T, Tamura T, Okada Y, Mikami H. Measurements of ligamentum flavum thickening at lumbar spine using MRI. Arch Orthop Trauma Surg 2009; 129:1415-9.

9. Abbas J, Hamoud K, Masharawi YM, et al. Ligamentum flavum thickness in normal and stenotic lumbar spines. Spine (Phila Pa 1976) 2010; 35:1225-30
10. Akreyi HA, Awdish HY. Assessment of ligamentum flavum thickness correlation with demographic variables and disc degeneration in Erbil governorate population sample. Zanco J.Med.Sci.2012; 16 :(2)145150

11. Kolte VS, Khambatta S, Ambiye MV. Thickness of the ligamenum flavum: Corelation with age and its asymmetry-A magnetic resonance imaging study. Asian Spine J 2015; 9(2):245-253. http://dx.doi.org/10.4184/asj.2015.9.2.245

12. Fukuyama $S$, Nakamura $T$, Ikeda $T$, Takagi K. The effect of mechanical stress on hypertrophy of the lumbar ligamentum flavum . J Spinal Disord 1995; 8:126- 30.

13. Okuda T, Baba I, Fujimoto Y et al. The pathology of ligamentum flavum in degenerative lumbar spine. Spine 2004; 29:1689-97

14. Grenier N, Kressel HY, Schiebler ML, et al. Normal and degenerative posterior spinal structures: MR imaging. Radiology 1987; 165:517-25.

15. Park JB, Chang H, Lee JK. Quantitative analysis of transforming growth factor-beta 1 in ligamentum flavum of lumbar spinal stenosis and disc herniation. Spine (Phila Pa 1976)2001;26: E492-95.

16. Chokshi FH, Quencer RM, Smoker WRK. The "thickened" Ligamentum Flavum: Is It Buckling or Enlargement? AJNR Am J Neuroradiol 2010; 31:181316.

17. Spurling R, Mayfield F, Rogers J. Hypertrophy of the ligament flava as a cause of low back pain. JAMA 1937;109:928

18. Horwitz T. Lesions of the intervertebral disc and ligamentum flavum of the lumbar vertebrae: anatomic study of 75 human cadavers. Surgery 1939; 6: 410-25

19. Ramani PS, Perry RH, Tomlinson BE. Role of ligamentum flavum in the symptomatology of prolapsed lumbar intervertebral discs. J Neurol Neurosurg Psychiatry 1975; 38: 550-7

20. Eiman M, Abdel-Menguid. An anatomical study of the human lumbar ligamentum flavum. Neurosciences 2008;13(1):11-16

21. Kouwenhowen JW, Vineken KL, Bartels LW et al. Analysis of pre existent vertebral rotation in the normal spine. Spine 2006; 31:1467-72.

\section{How to cite this article:}

Vijaya Rajesh Kamble (2017) ' Magnetic resonance imaging assessment of thickened ligamentum flavum in degenerative lumbar spine-corelation with age, gender, degenerative disc, right-left asymmetry in central india population', International Journal of Current Advanced Research, 06(03), pp. 2520-2523.

DOI: http://dx.doi.org/10.24327/ijcar.2017.2523.0041 